\title{
The Association of Anti-Aminoacyl-Transfer Ribonucleic Acid Synthetase Antibodies in Patients With Rheumatoid Arthritis and Interstitial Lung Disease
}

\author{
Masakazu MATSUSHITA, Naoto TAMURA, Michihiro OGASAWARA, \\ Kurisu TADA, Ken YAMAJI, Yoshinari TAKASAKI \\ Department of Internal Medicine and Rheumatology, Juntendo University, School of Medicine, Tokyo, Japan
}

\begin{abstract}
Objectives: This study aims to analyze the distribution and clinicopathological characteristics of anti-aminoacyl-transfer ribonucleic acid (tRNA) synthetase (ARS) antibodies in rheumatoid arthritis patients.

Patients and methods: We retrospectively studied the anti-ARS antibody levels in 228 RA patients' (44 males, 184 females; mean age $62.9 \pm 14.0$ years; range 23 to 88 years) sera from their medical charts. We determined the association with anti-cyclic citrullinated peptide antibody levels, interstitial lung disease (ILD), rheumatoid factor, and methotrexate or biological disease modifying antirheumatic drug treatments.

Results: Anti-ARS antibodies were detected in 14 RA patients (6.1\%). ILD complications were significantly higher among anti-ARS antibody-positive patients $(57.1 \%$ vs $22.4 \%, p<0.05)$. Levels of anti-threonyl-tRNA-synthetase (anti-PL-7) and anti-alanyl-tRNA-synthetase (anti-PL-12), two anti-ARS antibodies, were higher in RA patients with concurrent ILD (both $p<0.05$ ). Myositis and ILD worsening were not observed in three anti-ARS antibodypositive patients despite biological disease modifying antirheumatic drug administration. There was no difference in anti-cyclic citrullinated peptide and rheumatoid factor specificities between patients with or without ARS antibodies.

Conclusion: Anti-ARS antibodies were detected in RA patients, with higher prevalence in patients with concurrent ILD. RA patients, specifically those with ILD complications, should be tested for anti-ARS antibodies.

Keywords: Aminoacyl-transfer ribonucleic acid synthetase; antirheumatic drugs; interstitial lung diseases; rheumatoid arthritis.
\end{abstract}

A number of autoantibodies were detected in patients with connective tissue disorders., ${ }^{1,2}$ Disease severity often correlates with a change in antibody levels, and elevated levels of such autoantibodies can be used as a prognostic indicator for organ-related complications and therapeutic effectiveness. In 1980, Nishikai et al. ${ }^{3}$ first reported the specific association of anti-Jo-1 antibody in patients with polymyositis or dermatomyositis (PM/DM). This antibody was classified as myositis-specific autoantibody and its corresponding antigen was reported to be a $50-\mathrm{kDa}$ histidine transfer ribonucleic acid (tRNA) synthetase, a type of cytoplasmic aminoacyl-tRNA synthetase (ARS). ${ }^{4-6}$
ARS is an enzyme that attaches specific amino acids to tRNA in the presence of adenosine triphosphate, catalyzing the ARS reaction. Eight types of anti-ARS antibodies have been thus far identified. ${ }^{7}$ They have been found to be prevalent in arthritis and interstitial lung disease (ILD), skin lesions, and idiopathic interstitial pneumonia. ${ }^{8-11}$

Several studies on anti-ARS antibodies in $\mathrm{PM} / \mathrm{DM}$ patients have been conducted and their correlation with certain clinical presentations has been shown. ${ }^{7,12}$ However, limited information is available on the incidence of anti-ARS antibodies in other connective tissue disorders. Therefore, 
in this study, we aimed to analyze the distribution and clinicopathological characteristics of anti-ARS antibodies in rheumatoid arthritis (RA) patients.

\section{PATIENTS AND METHODS}

The study included a total of 228 RA patients

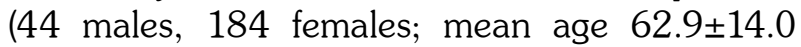
years; range 23 to 88 years) who visited the Juntendo University Hospital between April 2010 and March 2014. Standards proposed by the 2010 American College of Rheumatology/ European League Against Rheumatism were used for the diagnosis of RA. ${ }^{13}$ Autoimmune conditions were diagnosed based on the classification criteria for myositis. ${ }^{14}$ and ILD was confirmed by histopathology or by the detection of interstitial infiltrates after chest radiography (X-ray generator, 0.6/1.2 P324DK-125, Shimadzu Corporation, Kyoto, Japan, and X-ray photoreceiver, flat panel detector CALNEO-U, Fujifilm Medical, Tokyo, Japan) or high-resolution computed tomography (CT, Aquilion ONE TSX-301C/3A, Toshiba Medical Systems Corporation, Otawara City, Tochigi-ken Japan). The study protocol was approved by the Juntendo University Hospital Ethics Committee. A written informed consent was obtained from each patient. The study was conducted in accordance with the principles of the Declaration of Helsinki.

Anti-ARS antibodies were assessed using the line blot test kit (Myositis Profile Euroline Blot test kit, Euroimmun AG, Lübeck, Germany). The following antibodies were assayed: antiOJ (anti-isoleucyl) antibody against isoleucyltRNA synthetase; anti-EJ (anti-glycyl) antibody against glycyl-tRNA synthetase; anti-threonyltRNA synthetase (anti-PL7) antibody against threonyl-tRNA synthetase; anti-alanyl-tRNA synthetase (anti-PL-12) antibody against alanyltRNA synthetase; and anti-Jo-1 antibody against histidyl-tRNA synthetase. The manufacturer's protocols were followed for each measurement.

Sera from 14 patients who tested positive for anti-ARS antibodies were screened for anti-cytoplasmic antibody using indirect immunofluorescence test and commercially available human epithelial type 2 monolayer slides (Medical \& Biological Laboratories, Co.,
Ltd., Nagoya, Japan). The sera were diluted 1:100 in phosphate-buffered saline (PBS) and incubated at room temperature for 45 minutes. After incubation, the slides were rinsed in PBS, followed by incubation with fluorescein isothiocyanateconjugated goat anti-human immunoglobulin G (KPL, Gaithersburg, MD, USA) diluted 1:50 in PBS as a secondary detecting reagent, for 45 minutes at room temperature. After washing with PBS, the staining pattern was examined.

A commercial second-generation enzymelinked immunosorbent assay kit (Immunoscan RA anti-cyclic citrullinated peptide [anti-CCP] test kit; Euro Diagnostica, Malmö, Sweden) was used to detect anti-CCP antibodies, following manufacturer's instructions. Anti-CCP antibody was measured by the laboratory test company (SRL Inc., Hino-shi, Tokyo, Japan; test code 3301D). Briefly, serum samples were transferred to microtiter plates after a 1:50 dilution, and incubated at room temperature for 60 minutes. After washing, the plates were incubated with peroxidase-conjugated antihuman immunoglobulin $G$ antibody at room temperature for 30 minutes. Samples were incubated with 3,3', 5,5'-tetramethylbenzidine substrate solution at room temperature and the reaction was stopped with phosphoric acid after 30 minutes. Optical density measurements were conducted in a plate reader (Multiskan MS, Labsystems, Helsinki, Finland) at $450 \mathrm{~nm}$. Each plate contained pre-diluted anti-CCP standards, and positive and negative controls. Anti-CCP antibody levels in serum samples were analyzed by a four-parameter curve fit. Samples with values exceeding $25 \mathrm{U} / \mathrm{mL}$ were considered positive for anti-CCP antibodies.

Rheumatoid factor (RF) was measured using a semi-quantitative latex agglutination test. A commercial kit (RapiTex RF; Behring, Marburg, Germany) was used following the manufacturer's protocol. Serum titers greater than $1 / 4(80 \mathrm{IU} / \mathrm{mL})$ were considered RF-positive.

\section{Statistical analysis}

Fisher's exact test was applied to analyze statistical significance between groups using SPSS version 10 software (SPSS Inc., Chicago, IL, USA). $P$ values of $<0.05$ were considered statistically significant. 
Table 1. Demographics of rheumatoid arthritis subjects $(\mathrm{n}=228)$

\begin{tabular}{lccc}
\hline & $\mathrm{n}$ & $\%$ & Mean $\pm \mathrm{SD}$ \\
\hline Age (year) & & & $62.9 \pm 14.0$ \\
Sex & & & \\
$\quad$ Male & 44 & & \\
$\quad$ Female & 184 & & \\
MTX administration & 134 & 58.8 \\
bDMARDs administration & 96 & 42.1 \\
Patients with concurrent ILD (\%) & 56 & 24.6 \\
ILD, MTX-treated & 20 & 35.7 \\
ILD, bDMARDs-treated & 17 & 30.4 \\
SD: Standard deviation; MTX: Methotrexate; bDMARDs: Biological disease \\
modifying antirheumatic drugs; ILD: Interstitial lung disease.
\end{tabular}

\section{RESULTS}

Table 1 shows the demographics of the 228 RA patients included in this study. Anti-ARS antibodies were detected in 14 patients (6.1\%). Six of these patients had anti-PL-7 antibodies (2.6\%); four had anti-EJ antibodies (1.8\%); two had anti-PL-12 antibodies (0.9\%); one had anti-OJ antibodies (0.4\%); and one had anti-Jo-1 antibodies (0.4\%). No patient tested positive for two or more anti-ARS antibodies. Anti-nuclear indirect fluorescence test revealed cytoplasmic staining with sera of patients positive for ARS (Figure 1), suggesting their anti-ARS specificity.

Data were stratified as anti-ARS antibodypositive and anti-ARS antibody-negative (Table 2). No statistically significant difference was noted between anti-ARS antibody-negative and anti-ARS antibody-positive patients in terms of age, sex, methotrexate (MTX) administration frequency, biological disease modifying antirheumatic drugs
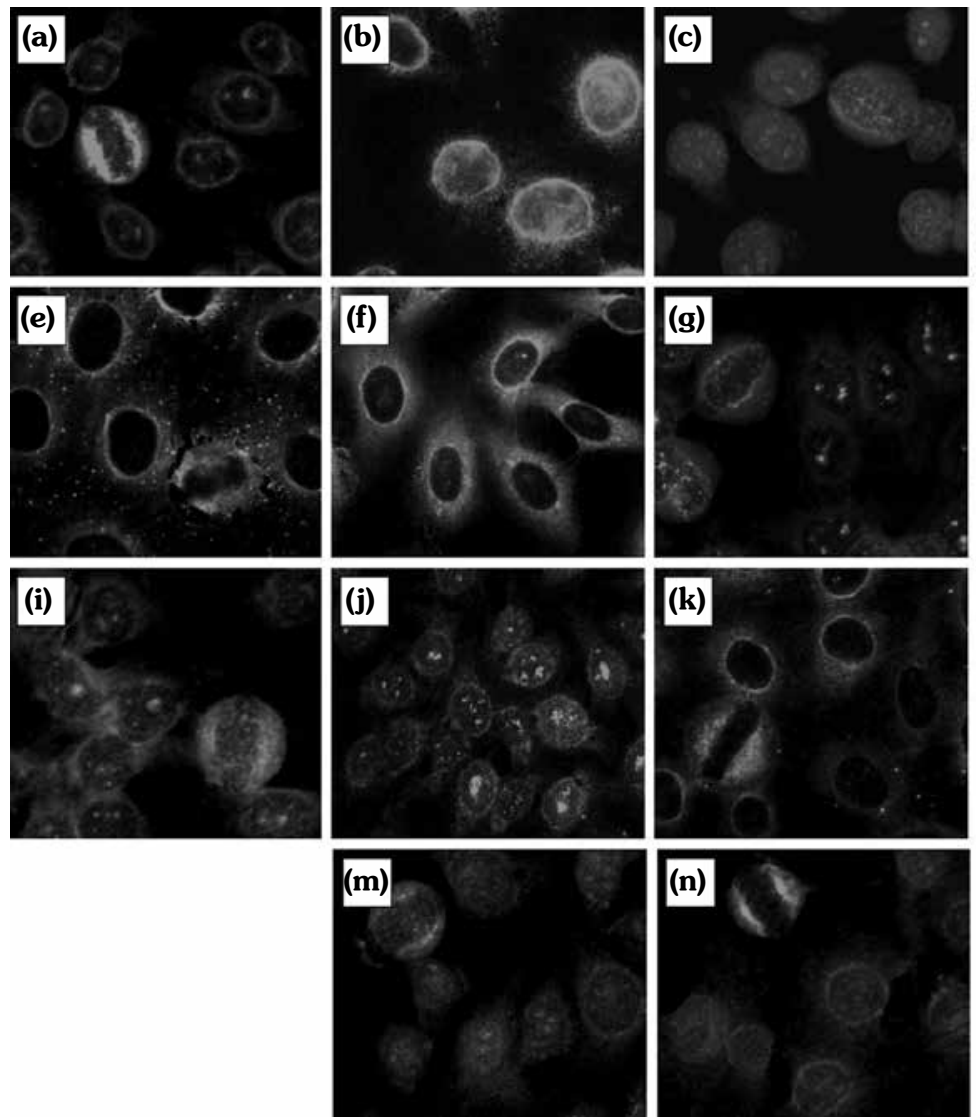
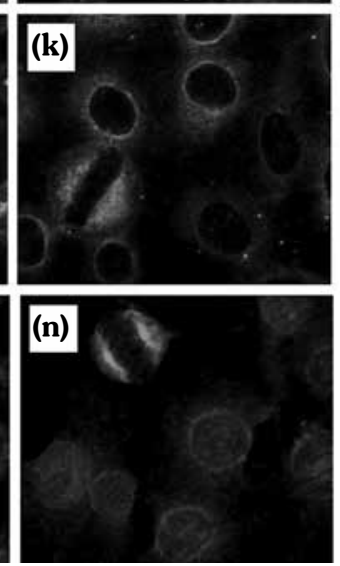
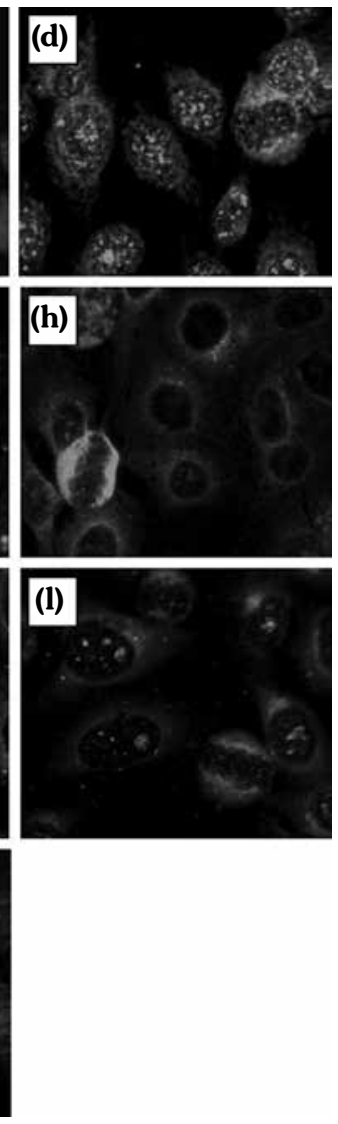

Figure 1. Anti-nuclear antibody (indirect fluorescence) test. Test was performed using human epithelial type 2 to verify cytoplasmic reactivity of anti-aminoacyl-transfer ribonucleic acid synthetase antibodies. Patients 1-6 had anti-PL-7 antibodies (a-f); patients 7-10 had anti-EJ antibodies (g-j); patients 11 and 12 had anti-PL-12 antibodies (k and $\mathbf{l})$; and patients 13 and 14 had anti-OJ (m) and anti-Jo-1 (n) antibodies, respectively. 
Table 2. Comparison of anti-aminoacyl-transfer ribonucleic acid synthetase antibody-positive and -negative patients

\begin{tabular}{|c|c|c|c|c|c|c|c|}
\hline & \multicolumn{3}{|c|}{ Anti-ARS antibody-positive } & \multicolumn{3}{|c|}{ Anti-ARS antibody-negative } & \multirow[b]{2}{*}{$p$} \\
\hline & $\mathrm{n}$ & $\%$ & Mean \pm SD & $\mathrm{n}$ & $\%$ & Mean \pm SD & \\
\hline Age (year) & & & $65.0 \pm 13.6$ & & & $62.8 \pm 14.0$ & NS \\
\hline Sex & & & & & & & NS \\
\hline Male & 1 & & & 1 & & & \\
\hline Female & 2.5 & & & 4.5 & & & \\
\hline Interstitial lung disease (+) & 8 & 57.1 & & 48 & 22.4 & & $<0.05$ \\
\hline Methotrexate (+) & 4 & 28.6 & & 130 & 60.7 & & NS \\
\hline bDMARDs (+) & 3 & 21.4 & & 92 & 43.0 & & NS \\
\hline Rheumatoid factor $(\mathrm{U} / \mathrm{mL})$ & & & $221.5 \pm 401.1$ & & & $122.7 \pm 306.9$ & NS \\
\hline Rheumatoid factor-positive rate & & 66.7 & & & 57.3 & & NS \\
\hline Anti-CCP $(\mathrm{U} / \mathrm{mL})$ & & & $214.5 \pm 314.0$ & & & $271.6 \pm 686.7$ & NS \\
\hline Anti-CCP-positive rate & & 85.7 & & & 78.3 & & NS \\
\hline
\end{tabular}

(bDMARDs) administration frequency, RF values, and anti-CCP levels. However, ILD complications were significantly more frequent in the anti-ARS antibody-positive group than in the negative group (57.1\% vs. $22.4 \%, p<0.05)$. To understand the effect of ILD on the prevalent antibody type, data were further stratified with respect to patient ILD status (Table 3). The incidence of anti-ARS antibody positivity was significantly higher in the ILD group $(14.3 \%$ vs. $3.5 \%, \mathrm{p}<0.05)$ and a significantly higher percentage of RA patients with ILD tested positive for anti-PL-7 antibody and anti-PL-12 antibody than non-ILD RA patients. No statistically significant differences were noted for other antibodies.

Table 4 contains detailed information (anti-ARS antibody status, age, sex, MTX and bDMARDs treatments, anti-CCP, and RF values) for patients

Table 3. Anti-aminoacyl-transfer ribonucleic acid synthetase antibodies in rheumatoid arthritis patients according to interstitial lung disease status

\begin{tabular}{|c|c|c|c|c|c|}
\hline & \multicolumn{2}{|c|}{$\begin{array}{l}\text { Patients with } \\
\text { concurrent ILD (\%) }(\mathrm{n}=56)\end{array}$} & \multicolumn{2}{|c|}{$\begin{array}{l}\text { Patients without } \\
\text { concurrent ILD }(\%)(n=172)\end{array}$} & \multirow[b]{2}{*}{$p$} \\
\hline & $\mathrm{n}$ & $\%$ & $\mathrm{n}$ & $\%$ & \\
\hline Anti-ARS antibody (+) & 8 & 14.3 & 6 & 3.5 & $<0.05$ \\
\hline Anti-PL-7 antibody & 4 & 7.1 & 2 & 1.2 & $<0.05$ \\
\hline Anti-EJ antibody & 2 & 3.6 & 2 & 1.2 & NS \\
\hline Anti-PL-12 antibody & 2 & 3.6 & 0 & 0.0 & $<0.05$ \\
\hline Anti-OJ antibody & 0 & 0.0 & 1 & 0.6 & NS \\
\hline Anti-Jo-1 antibody & 0 & 0.0 & 1 & 0.6 & NS \\
\hline
\end{tabular}

Table 4. Anti-aminoacyl-transfer ribonucleic acid synthetase antibody-positive rheumatoid arthritis patients with interstitial lung disease

\begin{tabular}{ccccccc}
\hline Patients & Anti-ARS & Age/Sex & MTX & bDMARDs & Anti-CCP (U/mL) & RF (U/mL) \\
\hline 1 & PL-7 & $63 / \mathrm{M}$ & - & ABA & 364 & 1419 \\
2 & & $79 / \mathrm{F}$ & $2 \mathrm{mg} / \mathrm{W}$ & - & 198 & 96 \\
3 & & $80 / \mathrm{F}$ & - & - & $<0.6$ & $<3$ \\
4 & & $54 / \mathrm{F}$ & - & - & 228 & 61 \\
5 & PL-12 & $37 / \mathrm{M}$ & - & ABA & 349 & 18.4 \\
6 & & $51 / \mathrm{F}$ & - & ETN & 1110 & 127 \\
7 & EJ & $75 / \mathrm{F}$ & - & - & 319 & 727 \\
8 & & $88 / \mathrm{F}$ & - & - & 5.6 & 135 \\
\hline
\end{tabular}

ARS: Aminoacyl-transfer ribonucleic acid synthetase; MTX: Methotrexate; bDMARDs: Biological disease modifying antirheumatic drugs; Anti-CCP: Anti-cyclic citrullinated peptide; RF: Rheumatoid factor; EJ: Anti-glycyl; ABA: Abatacept; ETN: Etanercept. 

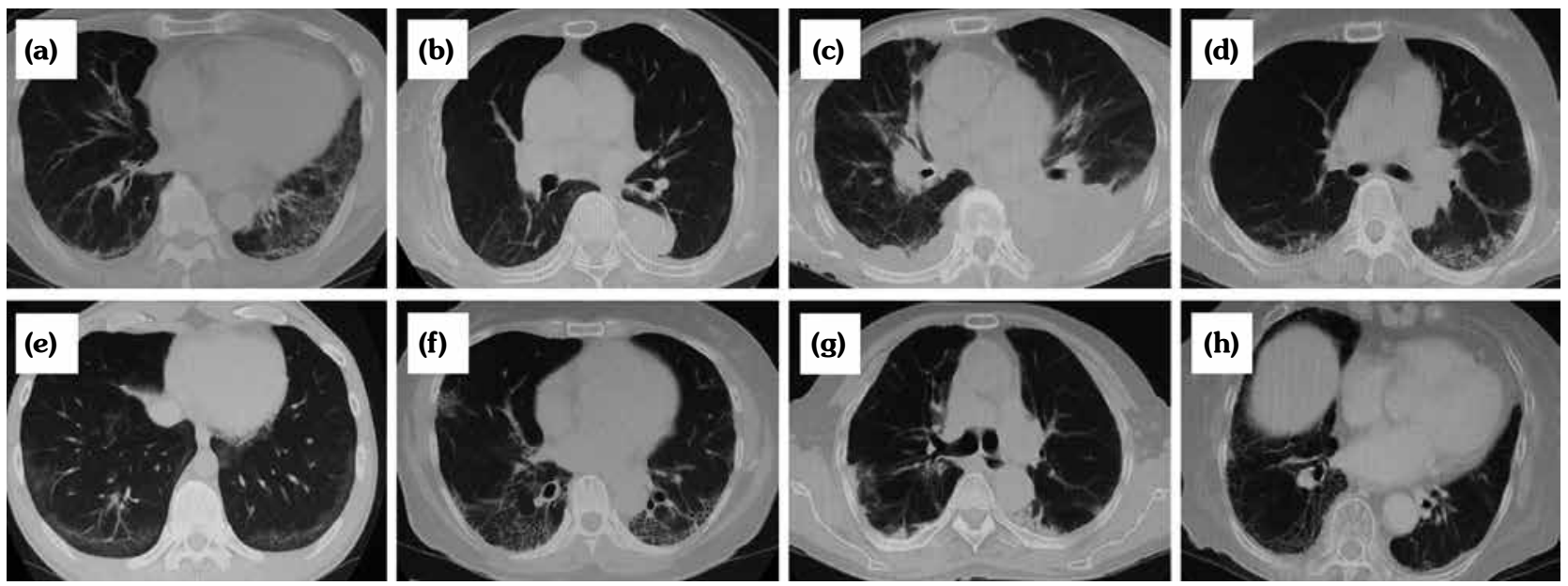

Figure 2. Chest computed tomography findings relative to patients testing positive for anti-aminoacyl-transfer ribonucleic acid synthetase antibodies and with concurrent non-specific interstitial lung disease. Patients 1-4 had anti-PL-7 antibodies (a-d); patients 5 and 6 had anti-PL-12 antibodies (e and f, respectively); and patients 7 and 8 had anti-EJ antibodies (g and h, respectively).

who tested positive for anti-ARS antibody and had also ILD. MTX was administered to one patient while bDMARDs were administered to three patients. Abatacept and etanercept were administered to two and one patients, respectively. Except for one patient who tested positive for anti-PL-7 antibody, anti-CCP antibody and RF titers were high. Chest $\mathrm{CT}$ revealed that these patients did not have PM/DM during the clinical course of the disease (Figure 2). Chest CT scans revealed non-specific interstitial pneumonia lung complications in all patients. No characteristic changes were associated with the respective anti-ARS antibodies detected in patient sera. Moreover, patients with rapid progression of lung complications were absent.

\section{DISCUSSION}

Our study revealed the presence of anti-ARS antibodies in sera of $6.1 \%$ RA patients in our cohort; $57.1 \%$ of anti-ARS antibody-positive RA patients had concomitant ILD. This is a very significant result since, generally, anti-ARS antibodies are detected in PM/DM patients but not in RA patients. Notably, RA patients testing positive for anti-ARS antibodies did not differ from RA patients testing negative for these antibodies in terms of age, sex, RF positivity, and anti-CCP antibody positivity. However, the incidence of ILD complications was significantly higher in RA patients who were positive for anti-ARS antibodies in comparison with patients who tested negative, and CT scan findings from all ILD patients were suggestive of non-specific interstitial pneumonia.

Anti-ARS antibodies are known as myositisspecific autoantibodies and have been extensively detected in idiopathic inflammatory myopathies ${ }^{15}$ and in patients with inflammatory myopathies overlapping with RA. ${ }^{16}$ Bernstein et al. $^{6}$ have reported anti-Jo-1 antibodies in the sera of $25 \%$ of $\mathrm{PM} / \mathrm{DM}$ patients, $7.5 \%$ of myositis patients without the complication of ILD, $68 \%$ of patients with ILD complication, and 3\% of patients with ILD in the absence of myositis. In another study, anti-Jo-1 antibodies have also been reported in $15-30 \%$ of PM patients and $60-70 \%$ of ILD patients. ${ }^{17}$ Other anti-ARS antibodies are less predominant in inflammatory myopathies. In our study, however, anti-Jo-1 antibody was detected in only one RA patient, whereas anti-PL-7 antibody was detected in six patients (42.9\% of all anti-ARS antibody-positive patients). Early diagnosis of these antibodies has been associated with a better overall survival. ${ }^{18-20}$

In RA patients with concomitant ILD, PL-7, PL-12, and EJ antibodies were detected, with PL-7 antibodies having the highest incidence. Targoff et al. ${ }^{21}$ reported a correlation 
between anti-EJ antibody incidence and ILD in PM patients, and established that these antibodies reacted with glycyl-tRNA synthetase. However, anti-PL-7, anti-PL-12, anti-OJ, anti-Zo (anti-phenylalanyl), and anti-KS (antiasparaginyl) antibodies were reported to have a stronger correlation with ILD than that between anti-Jo-1 antibody and DM/PM patients. ${ }^{22-27}$ In our population of RA patients, anti-PL-7 antibodies were most frequently detected in patients who had ILD complications (four); PL-12 and EJ antibodies were each detected in two patients. Notably, however, the anti-PL-12 antibody was detected only among ILD patients. Kalluri et al. ${ }^{28}$ reported that $90 \%$ of patients who tested positive for anti-PL-12 antibodies also had ILD. They have concluded that the presence of anti-PL-12 antibodies is more strongly associated with ILD than with myositis and arthritis. ${ }^{25}$

Biological disease modifying antirheumatic drugs, such as tumor necrosis factor, interleukin-6, or cytotoxic T-lymphocyte associated protein 4, are recommended for the treatment of RA and they play an important role in improving the patient's activities of daily living and quality of life. $^{28}$ Recent reports suggest that anti-tumor necrosis factor-alpha treatment of patients with elevated anti-ARS antibody levels may trigger myositis. ${ }^{29}$ However, our results show that even in RA patients testing positive for anti-ARS antibodies and who were administered bDMARDs, connective tissue disorder complications such as $\mathrm{PM} / \mathrm{DM}$ or transition/progression were absent. Although ILD complication was observed in 57\% of RA patients who had autoantibodies against ARS, treatment with MTX and bDMARDs could be safely and effectively administered. Moreover, rapid progression of ILD was not seen in myositis patients testing positive for anti-ARS antibodies. ${ }^{30}$

Our study has certain limitations. First, being a retrospective study, the comprehensiveness of data collection could not be ascertained; second, this is a single-center study, which might be subject to regional bias and limit our sample size. Additional multicenter large-scale prospective studies are required to establish the anti-ARS antibody profile of RA patients. Furthermore, as we were unable to analyze the levels of antiARS antibodies in healthy subjects, a more controlled study is desired. We have, however, found that patients with other diseases such as
Sjögren's syndrome and microscopic polyangiitis are negative for anti-ARS antibodies. In spite of these limitations, the results of the study are important because, for the first time, they provide strong evidence of anti-ARS antibodies in RA patients, with a different distribution between ILD-positive and -negative patients.

In conclusion, line blot analysis of anti-ARS antibody levels in RA patients revealed that these autoantibodies were present in $6 \%$ of RA patients. Autoantibodies, particularly anti-PL-7 and anti PL-12 antibodies, were detected in a significantly higher percentage in patients with the complication of ILD. No significant effects of bDMARDs on the incidence of connective tissue disorder complications/transition/progression were observed in anti-ARS antibody-positive RA patients. RA patients, specifically those with ILD complications, should be tested for Anti-ARS antibodies. Ultimately, we hope that the results of this study will have therapeutic and diagnostic implications and will stimulate further research in this important area.

\section{Declaration of conflicting interests}

The authors declared no conflicts of interest with respect to the authorship and/or publication of this article.

\section{Funding}

The authors received no financial support for the research and/or authorship of this article.

\section{REFERENCES}

1. Myckatyn SO, Russell AS. Outcome of positive antinuclear antibodies in individuals without connective tissue disease. J Rheumatol 2003;30:736-9.

2. Bombardieri S, Neri R, Tartarelli G, d'Ascanio A, Giovanelli L. The clinical relevance of antinuclear antibodies in connective tissue diseases. Scand $\mathrm{J}$ Rheumatol Suppl 1987;66:35-45.

3. Nishikai M, Reichlin M. Heterogeneity of precipitating antibodies in polymyositis and dermatomyositis. Characterization of the Jo- 1 antibody system. Arthritis Rheum 1980;23:881-8.

4. Rosa MD, Hendrick JP Jr, Lerner MR, Steitz JA, Reichlin M. A mammalian tRNAHis-containing antigen is recognized by the polymyositis-specific antibody antiJo-1. Nucleic Acids Res 1983;11:853-70.

5. Mathews MB, Bernstein RM. Myositis autoantibody inhibits histidyl-tRNA synthetase: a model for autoimmunity. Nature 1983;304:177-9. 
6. Bernstein RM, Morgan SH, Chapman J, Bunn CC, Mathews MB, Turner-Warwick M, et al. Anti-Jo-1 antibody: a marker for myositis with interstitial lung disease. Br Med J (Clin Res Ed) 1984;289:151-2.

7. Satoh M, Tanaka S, Ceribelli A, Calise SJ, Chan EK. A Comprehensive Overview on Myositis-Specific Antibodies: New and Old Biomarkers in Idiopathic Inflammatory Myopathy. Clin Rev Allergy Immunol 2017;52:1-19.

8. Hirakata M, Suwa A, Nagai S, Kron MA, Trieu $E P$, Mimori $T$, et al. Anti-KS: identification of autoantibodies to asparaginyl-transfer RNA synthetase associated with interstitial lung disease. $\mathrm{J}$ Immunol 1999;162:2315-20.

9. Okayasu K, Ohtani Y, Takemura T, Uchibori K, Tamaoka M, Furuiye M, et al. Nonspecific interstitial pneumonia (NSIP) associated with anti-KS antibody: differentiation from idiopathic NSIP. Intern Med 2009;48:1301-6.

10. Rönnelid J, Barbasso Helmers S, Storfors H, Grip K, Rönnblom L, Franck-Larsson K, et al. Use of a commercial line blot assay as a screening test for autoantibodies in inflammatory myopathies. Autoimmun Rev 2009;9:58-61.

11. Ghirardello A, Rampudda M, Ekholm L, Bassi N, Tarricone E, Zampieri S, et al. Diagnostic performance and validation of autoantibody testing in myositis by a commercial line blot assay. Rheumatology (Oxford) 2010;49:2370-4.

12. Hozumi $\mathrm{H}$, Enomoto $\mathrm{N}$, Kono $\mathrm{M}$, Fujisawa $\mathrm{T}$, Inui $\mathrm{N}$, Nakamura $\mathrm{Y}$, et al. Prognostic significance of anti-aminoacyl-tRNA synthetase antibodies in polymyositis/dermatomyositis-associated interstitial lung disease: a retrospective case control study. PLoS One 2015;10:e0120313.

13. Aletaha D, Neogi T, Silman AJ, Funovits J, Felson DT, Bingham CO, et al. 2010 Rheumatoid arthritis classification criteria: an American College of Rheumatology/European League Against Rheumatism collaborative initiative. Arthritis Rheum 2010;62:2569-81.

14. Bohan A, Peter JB. Polymyositis and dermatomyositis (first of two parts). N Engl J Med 1975;292:344-7.

15. Mahler M, Miller FW, Fritzler MJ. Idiopathic inflammatory myopathies and the anti-synthetase syndrome: a comprehensive review. Autoimmun Rev 2014;13:367-71.

16. Nakajima A, Yoshino K, Soejima M, Kawaguchi $\mathrm{Y}$, Satoh T, Kuwana M, et al. High frequencies and co-existing of myositis-specific autoantibodies in patients with idiopathic inflammatory myopathies overlapped to rheumatoid arthritis. Rheumatol Int 2012;32:2057-61.

17. Mahler M, Fritzler MJ. Epitope specificity and significance in systemic autoimmune diseases. Ann N Y Acad Sci 2010;1183:267-87.
18. Cavagna L, Nuño L, Scirè CA, Govoni M, Longo FJ, Franceschini $F$, et al. Clinical Spectrum Time Course in Anti Jo-1 Positive Antisynthetase Syndrome: Results From an International Retrospective Multicenter Study. Medicine (Baltimore) 2015;94:1144.

19. Tillie-Leblond I, Wislez M, Valeyre D, Crestani B, Rabbat A, Israel-Biet $\mathrm{D}$, et al. Interstitial lung disease and anti-Jo-1 antibodies: difference between acute and gradual onset. Thorax 2008;63:53-9.

20. Aggarwal R, Cassidy E, Fertig N, Koontz DC, Lucas M, Ascherman DP, et al. Patients with nonJo-1 anti-tRNA-synthetase autoantibodies have worse survival than Jo-1 positive patients. Ann Rheum Dis 2014;73:227-32.

21. Targoff IN, Trieu EP, Plotz PH, Miller FW. Antibodies to glycyl-transfer RNA synthetase in patients with myositis and interstitial lung disease. Arthritis Rheum 1992;35:821-30.

22. Targoff IN, Arnett FC, Reichlin M. Antibody to threonyl-transfer RNA synthetase in myositis sera. Arthritis Rheum 1988;31:515-24.

23. Sato S, Hirakata M, Kuwana M, Nakamura K, Suwa A, Inada S, et al. Clinical characteristics of Japanese patients with anti-PL-7 (anti-threonyl-tRNA synthetase) autoantibodies. Clin Exp Rheumatol 2005;23:609-15.

24. Hirakata M, Suwa A, Takada T, Sato S, Nagai S, Genth E, et al. Clinical and immunogenetic features of patients with autoantibodies to asparaginyltransfer RNA synthetase. Arthritis Rheum 2007;56:1295-303.

25. Targoff IN, Arnett FC. Clinical manifestations in patients with antibody to PL-12 antigen (alanyl-tRNA synthetase). Am J Med 1990;88:241-51.

26. Sato S, Kuwana M, Hirakata M. Clinical characteristics of Japanese patients with anti-OJ (anti-isoleucyl-tRNA synthetase) autoantibodies. Rheumatology (Oxford) 2007;46:842-5.

27. Betteridge Z, Gunawardena H, North J, Slinn $\mathrm{J}$, McHugh N. Anti-synthetase syndrome: a new autoantibody to phenylalanyl transfer RNA synthetase (anti-Zo) associated with polymyositis and interstitial pneumonia. Rheumatology (Oxford) 2007;46:1005-8.

28. Kalluri M, Sahn SA, Oddis CV, Gharib SL, Christopher-Stine L, Danoff SK, et al. Clinical profile of anti-PL-12 autoantibody. Cohort study and review of the literature. Chest 2009;135:1550-6.

29. Ishikawa Y, Yukawa N, Kawabata D, Ohmura K, Fujii $\mathrm{T}$, Usui $\mathrm{T}$, et al. A case of antisynthetase syndrome in a rheumatoid arthritis patient with anti-PL-12 antibody following treatment with etanercept. Clin Rheumatol 2011;30:429-32.

30. Hallowell RW, Danoff SK. Interstitial lung disease associated with the idiopathic inflammatory myopathies and the antisynthetase syndrome: recent advances. Curr Opin Rheumatol 2014;26:684-9. 